\title{
Existence and multiplicity results for some nonlinear problems with singular $\phi$-Laplacian via a geometric approach
}

\author{
Xuelei Wang ${ }^{1,2}$, Qihuai Liü ${ }^{3 *}$ and Dingbian Qian
}

\section{"Correspondence:}

qhuailiu@gmail.com

${ }^{3}$ School of Mathematics and

Computing Sciences, Guilin

University of Electronic Technology,

Guilin, 541002, China

Full list of author information is

available at the end of the article

\begin{abstract}
New results on the existence and multiplicity of the solutions for some nonlinear boundary value problems with singular $\phi$-Laplacian are obtained via a bend-twist fixed point theorem. These results improve related theorems in the previous literature. Moreover, the geometric approach in this paper provides a new method to investigate the existence and multiplicity of periodic motions of charged particles in a three-dimensional electromagnetic field.
\end{abstract}

Keywords: periodic solution; geometric approach; relativistic oscillator; singular $\phi$-Laplacian

\section{Introduction}

In this paper, we are concerned with the existence and multiplicity of the solutions of boundary value problems for nonlinear differential equations with the vectorial singular $\phi$-Laplacian

$$
\left(\phi\left(\mathrm{x}^{\prime}\right)\right)^{\prime}=\mathrm{f}\left(t, \mathrm{x}, \mathrm{x}^{\prime}\right)
$$

where $\mathrm{x} \in \mathbb{R}^{3}$, and $\phi: B_{a}(0) \rightarrow \mathbb{R}^{3}$ is a homeomorphism of the open ball $B_{a}(0)$ centered at the origin with the radius $a(0<a<+\infty)$ such that $\phi(0)=0$ and $|\phi(\mathrm{v})| \rightarrow \infty$ as $v \rightarrow$ $\partial B_{a}(0), \phi_{i}(i=1,2,3)$ is monotonous with respect to the $i$ th component of variable, and $\mathrm{f}(t, \mathrm{u}, \mathrm{v}):[0, T] \times \mathbb{R}^{3} \times \mathbb{R}^{3} \rightarrow \mathbb{R}^{3}$ is a Lipschitz continuous vector-valued function.

Equation (1.1) includes many interesting physical and mechanical models. A significant example is that equation (1.1) describes the dynamics of a charged particle in electric and magnetic fields when the particle velocities are relativistic [1].

Let us consider a particle of mass $m>0$ and charge $e$ in an electromagnetic field, where the electric potential is $V(\mathrm{x})$, and the periodic magnetic vector potential $\mathrm{A}(t)$ depends on time $t$ periodically with the least period $T$. The position of the particle is given by $\mathrm{x} \in \mathbb{R}^{3}$. Then the relativistic equations of the motion of the particle are described by the Lagrange function

$$
\mathcal{L}(\mathrm{x}, \dot{\mathrm{x}}, t)=m c^{2}\left(1-\sqrt{1-\frac{|\dot{\mathrm{x}}|^{2}}{c^{2}}}\right)-e V(\mathrm{x})+\frac{e}{c}\langle\dot{\mathrm{x}}, \mathrm{A}(t)\rangle,
$$

(c) 2016 Wang et al. This article is distributed under the terms of the Creative Commons Attribution 4.0 International License (http://creativecommons.org/licenses/by/4.0/), which permits unrestricted use, distribution, and reproduction in any medium, provided you give appropriate credit to the original author(s) and the source, provide a link to the Creative Commons license, and indicate if changes were made. 
where $\langle\cdot, \cdot\rangle$ denotes the usual inner product, and $c$ is the speed of light. By the standard variational principle the function (1.2) leads to the Euler-Lagrange equation

$$
\frac{\mathrm{d}}{\mathrm{d} t} \mathcal{L}_{\dot{\mathrm{x}}}(\mathrm{x}, \dot{\mathrm{x}}, t)=\mathcal{L}_{\mathrm{x}}(\mathrm{x}, \dot{\mathrm{x}}, t)
$$

which is a system consisting of three second-order differential equations

$$
\frac{\mathrm{d}}{\mathrm{d} t}\left(\frac{m \dot{x_{i}}}{\sqrt{1-\frac{|\dot{\mathrm{x}}|^{2}}{c^{2}}}}\right)+V_{x_{i}}(\mathrm{x})+\frac{e}{c} A_{i}(t)=0, \quad i=1,2,3 .
$$

When the magnetic potential $A$ is independent of time $t$, equation (1.3) corresponds to an autonomous Hamiltonian system of second-order differential equations, which is discussed by Moser and Zehnder [2], pp.16-17. These problems were also studied by many mathematicians and physicists (see [3-7]).

In case that the electric and magnetic potentials are symmetric with respect to the threedimensional space, equation (1.1) drops into the one-dimensional system

$$
\left(\phi\left(x^{\prime}\right)\right)^{\prime}=f\left(t, x, x^{\prime}\right)
$$

where $\phi:(-a, a) \rightarrow \mathbb{R}(0<a<+\infty)$ is a monotonous homeomorphism such that $\phi(0)=0$, and $f:[0, T] \times \mathbb{R}^{2} \rightarrow \mathbb{R}$ is locally Lipschitz continuous function.

Recently, Bereanu and Mawhin obtained several interesting results on the existence and multiplicity of the solutions for various boundary value problems of equation (1.4) by using the Leray-Schauder degree, the lower and upper solutions, and the method of variational calculation; see [8-15] and other related works; we can refer to [16-19]. In particular, a universal existence theorem for Dirichlet problem and an existence theorem for periodic and Neumann problem were established when $f$ satisfies some sign conditions [8]. Moreover, the problem for the existence of periodic solutions under the Hartman-type condition or the modified Hartman-type condition was considered in [20].

A special interesting example of (1.4) is given by a one-dimensional singular $\phi$-Laplacian of relativistic type $\left(\phi(s)=\frac{s}{\sqrt{1-s^{2}}}\right)$, which describes the dynamics of the acceleration of a relativistic particle of mass one at rest moving on a straight line (with the velocity of light normalized to one) [21, 22]. The existence of periodic solutions for the forced pendulum equation with relativistic effects

$$
\left(\frac{x^{\prime}}{\sqrt{1-\frac{\left|x^{\prime}\right|^{2}}{c^{2}}}}\right)^{\prime}+k x^{\prime}+a \sin x=p(t)
$$

was proved by Torres [23], where $c>0$ is the speed of light in the vacuum, and $k \geq 0$ is a possible viscous friction coefficient. The result shows that if $2 c T \leq 1$, then for any values $a$, $k$ and any $p \in \widetilde{C}_{T}$, equation (1.5) has at least one $T$-periodic solution, where $\widetilde{C}_{T}$ denote the Banach space of continuous and $T$-periodic functions with zero mean value. The result in [23] was extended to generalized pendulum-type equation with Liénard term [24]

$$
\left(\phi\left(x^{\prime}\right)\right)^{\prime}+f(x) x^{\prime}+g(x)=e(t)+s .
$$


The method used in $[23,24]$ is based on a nonstandard change of variables (based on some sign condition of $g^{\prime}(x)$ ) and an application of the Schauder fixed point theorem. Recently, Littlewood's boundedness problem and the existence of quasi-periodic solutions for relativistic oscillators with anharmonic potentials were studied in [25].

The existence of periodic solutions for nonlinear problems with singular $\phi$-Laplacian was extended to the $n$-dimensional case by Brezis and Mawhin [26], where the approach is mostly variational, but requires the use of results on an auxiliary system based upon fixed point theory and Leray-Schauder degree. The multiplicity and existence of periodic solutions in the $n$-dimensional case was proved by Mawhin in [27] by using a LusternikSchnirelman-type multiplicity result for some indefinite functionals. We refer to [28, 29] for the related developments.

The purpose of this paper is to find some method to deal with the problem with higherdimensional system (1.1). Our discussion is based on a geometric idea by phase-space analysis. We show that when $\mathrm{f}$ satisfies some general condition, then the solutions of (1.1) have some 'bend-twist' property in the generalized phase-space. This observation inspires us constructing a bend-twist fixed point theorem to prove the existence and multiplicity of periodic solutions for three-dimensional system (1.1).

More specifically, we extend the universal existence theorem for Dirichlet problem and the existence theorem for periodic (or Neumann) problem in [8] to the existence and multiplicity theorem for three-dimensional system, respectively. Even for the scalar equation (1.6), an interesting feature of our result is that the existence of periodic solutions is independent of the condition of $g^{\prime}(x)$ and the damping coefficient $f$.

The paper is organized as follows. Section 2 is devoted to introducing some preliminary results with respect to equation (1.1). A geometric fixed theorem (Theorem 2.1) is proved by simple topological degree argument. This theorem is the basic tool used in this paper. In Section 3.1, we obtain a universal existence theorem for equation (1.1) with the Dirichlet condition, which generalizes the result in [8]. This is not the case for other boundary condition; an existence and multiplicity result (see Theorem 3.2) is proved in Section 3.2 when $f$ satisfies some local sign condition. Periodic motions of relativistic oscillators of charged particles in a three-dimensional electromagnetic field are investigated in Section 3.3. In Section 4, a new result on the existence and multiplicity of periodic solutions of generalized pendulum-type equations that does not need any information upon the differentiability of $g$ is obtained by the geometric approach.

\section{Bend-twist theorem and some preliminary results}

In this section, we first introduce a geometric fixed point theorem, which will be used frequently in the subsequent sections. The geometric fixed point theorem is a small variation of the Poincaré-Miranda theorem (see [30] for instance), which goes back to Poincaré (1883) and has been used many times in the study of boundary value problems and periodic solutions. For example, see a recent paper [31] and the references therein.

Consider a parallelotope $\mathcal{D}$ with $2 n$ faces, that is,

$$
\mathcal{D}=\left\{\left(x_{1}, x_{2}, \ldots, x_{n}\right) \in \mathbb{R}^{n}: \alpha_{i} \leq x_{i} \leq \beta_{i}, i=1,2, \ldots, n\right\},
$$


Figure 1 Bend-twist in various spaces: (a) a line segment in $\mathbb{R}^{1}$; (b) a rectangle in $\mathbb{R}^{2}$; (c) a cuboid in $\mathbb{R}^{3}$.

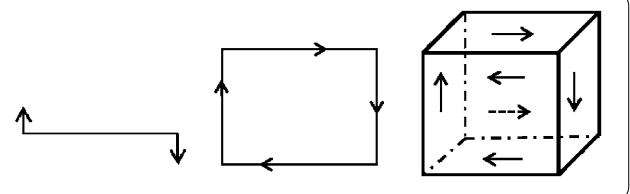

where $\alpha_{i}, \beta_{i}, i=1,2, \ldots, n$, are constants such that $\alpha_{i}<\beta_{i}$. The boundary of the parallelotope consists of $2 n$ faces. Denote them by

$$
\begin{aligned}
& V_{-}^{i}=\left\{\left(x_{1}, \ldots, x_{n}\right) \in \mathbb{R}^{n}: x_{i}=\alpha_{i}, \alpha_{j} \leq x_{j} \leq \beta_{j}, j \neq i \text { and } j \in\{1, \ldots, n\},\right. \\
& V_{+}^{i}=\left\{\left(x_{1}, \ldots, x_{n}\right) \in \mathbb{R}^{n}: x_{i}=\beta_{i}, \alpha_{j} \leq x_{j} \leq \beta_{j}, j \neq i \text { and } j \in\{1, \ldots, n\}\right\}
\end{aligned}
$$

respectively. Let $\Omega$ be an open subset of $\mathbb{R}^{n}$, and $\mathcal{D} \subset \Omega$. We say that a continuous map $\mathcal{F}=\left(\mathcal{F}_{1}, \mathcal{F}_{2}, \ldots, \mathcal{F}_{n}\right): \Omega \rightarrow \mathbb{R}^{n}$ satisfies the bend-twist condition on $\mathcal{D}$ if

$$
\mathcal{F}_{j_{i}}\left(V_{-}^{i}\right) \mathcal{F}_{j_{i}}\left(V_{+}^{i}\right) \leq 0, \quad i=1,2, \ldots, n,
$$

where $j_{i} \in\{1,2, \ldots, n\}$ are such that $j_{m} \neq j_{k}$ for $m \neq k$.

Here, $\mathcal{F}_{j_{i}}\left(V_{-}^{i}\right) \mathcal{F}_{j_{i}}\left(V_{+}^{i}\right) \leq 0$ means that $\mathcal{F}_{j_{i}}\left(V_{-}^{i}\right) \leq 0$ and $\mathcal{F}_{j_{i}}\left(V_{+}^{i}\right) \geq 0$, or $\mathcal{F}_{j_{i}}\left(V_{-}^{i}\right) \geq 0$ and $\mathcal{F}_{j_{i}}\left(V_{+}^{i}\right) \leq 0 ; \mathcal{F}_{j_{i}}\left(V_{ \pm}^{i}\right)>0$ means that $\mathcal{F}_{j_{i}}(\mathrm{x}) \geq 0$ for all $\mathrm{x} \in V_{ \pm}^{i}$ and there exists at least one $\mathrm{x}_{0} \in V_{ \pm}^{i}$ such that $\mathcal{F}_{j_{i}}\left(\mathrm{x}_{0}\right)>0 ; \mathcal{F}_{j_{i}}\left(V_{ \pm}^{i}\right)=0$ means that $\mathcal{F}_{j_{i}}(\mathrm{x})=0$ for all $\mathrm{x} \in V_{ \pm}^{i}$. The figures may help us to understand the 'bend-twist' condition well; see Figure 1.

Theorem 2.1 (Bend-twist theorem) Assume that a continuous map

$$
\mathcal{F}=\left(\mathcal{F}_{1}, \mathcal{F}_{2}, \ldots, \mathcal{F}_{n}\right): \Omega \rightarrow \mathbb{R}^{n}
$$

satisfies the bend-twist condition. Then there exists at least one point $\left(x_{0}^{1}, x_{0}^{2}, \ldots, x_{0}^{n}\right) \in \mathcal{D}$ such that $\mathcal{F}\left(x_{0}^{1}, x_{0}^{2}, \ldots, x_{0}^{n}\right)=0$.

Since the only difference between Theorem 2.1 and Poincaré-Miranda theorem is the permutation of the indexes in $\mathcal{F}$, that is, we have to consider $F_{j_{i}}$ instead of $F_{i}$, we omit the proof of Theorem 2.1. We can refer to [31] for an elementary proof based upon basic exterior calculus.

Next, we perform some preliminary results on the existence and uniqueness of a solution of initial value problem for equation (1.1) based on phase-plane analysis, which imply that the Poincaré mapping of (1.1) can be well defined.

Let $C_{T}$ denote the Banach space of continuous functions on $[0, T]$ with uniform norm $\|\cdot\|_{\infty}$, and $\widetilde{C}_{T}$ denote the Banach space of continuous and $T$-periodic functions with zero mean value. We also denote the inverse function of $\phi$ by $\phi^{-1}: \mathbb{R}^{3} \rightarrow B_{a}(0)(0<a<+\infty)$. Since $\phi_{i}(i=1,2,3)$ is a monotonous homeomorphism with respect to the $i$ th component of variable, the inverse function $\phi_{i}^{-1}$ is also a monotonous homeomorphism with respect to the $i$ th component of variable.

Lemma 2.1 Every solution of equation (1.1) can be uniquely defined on the interval $(-\infty,+\infty)$. 
Proof We rewrite equation (1.1) in the form

$$
x^{\prime}=\phi^{-1}(y), \quad y^{\prime}=f\left(t, x, \phi^{-1}(y)\right)
$$

Since $f:[0, T] \times \mathbb{R}^{3} \times \mathbb{R}^{3} \rightarrow \mathbb{R}^{3}$ is locally Lipschitz continuous, every solution of equation (2.1) with the initial value $\left(x_{0}, y_{0}\right)$ exists uniquely.

By the first equation of (2.1) and the bounded range of $\phi^{-1}$ we can see that if $x_{i}(t)$ exists, then

$$
\left|x_{i}^{\prime}(t)\right|<a .
$$

Hence, $|\mathrm{x}(t)|$ cannot go to infinity as $t$ goes to finite time. Moreover, the second equation of (2.1) implies that $\left|\mathrm{y}^{\prime}(t)\right|$ is bounded if $|\mathrm{x}(t)|$ is bounded. Therefore, $|\mathrm{y}(t)|$ cannot go to infinity as $t$ goes to finite time, that is, blow-up does not occur. The global existence of solutions is thus proved.

From Lemma 2.1 we can see that the Poincaré mapping of equation (1.1) is well defined. Let $\left(\mathrm{x}\left(t ; \mathrm{x}^{(0)}, \mathrm{y}^{(0)}\right), \mathrm{y}\left(t ; \mathrm{x}^{(0)}, \mathrm{y}^{(0)}\right)\right)$ be a solution of equation (2.1) with initial condition $\mathrm{x}\left(0 ; \mathrm{x}^{(0)}, \mathrm{y}^{(0)}\right)=\mathrm{x}^{(0)}, \mathrm{y}\left(0 ; \mathrm{x}^{(0)}, \mathrm{y}^{(0)}\right)=\mathrm{y}^{(0)}$. We define the Poincaré mapping $\mathcal{P}: \mathbb{R}^{3} \times \mathbb{R}^{3} \rightarrow$ $\mathbb{R}^{3} \times \mathbb{R}^{3}$ by

$$
\mathcal{P}\left(\mathrm{x}^{(0)}, \mathrm{y}^{(0)}\right)=\left(\mathrm{x}\left(T ; \mathrm{x}^{(0)}, \mathrm{y}^{(0)}\right), \mathrm{y}\left(T ; \mathrm{x}^{(0)}, \mathrm{y}^{(0)}\right)\right) .
$$

Consequently, $\mathcal{P}$ is a continuous and one-to-one mapping. Every zero point of the continuous mapping $\mathcal{F}: \mathbb{R}^{3} \times \mathbb{R}^{3} \rightarrow \mathbb{R}^{3} \times \mathbb{R}^{3}$, defined by $\mathcal{F}=\mathcal{P}$-id, corresponds to a $T$-periodic solution of equation (1.1), where id denotes the identity mapping.

\section{Three-dimensional systems with singular $\phi$-Laplacian}

\subsection{The case of Dirichlet boundary condition}

In this section, we investigate the existence of solutions of equation (1.1) with Dirichlet boundary condition

$$
\mathrm{x}(0)=0=\mathrm{x}(T) .
$$

A 'universal' solvability result is obtained, and we conclude the result in the following theorem.

Theorem 3.1 For each continuous vector-valued function $\mathrm{f}:[0, T] \times \mathbb{R}^{3} \times \mathbb{R}^{3} \rightarrow \mathbb{R}^{3}$, the Dirichlet boundary problem

$$
\left(\phi\left(\mathrm{x}^{\prime}\right)\right)^{\prime}=\mathrm{f}\left(t, \mathrm{x}, \mathrm{x}^{\prime}\right), \quad \mathrm{x}(0)=0=\mathrm{x}(T),
$$

has at least one solution.

In fact, the existence of Theorem 3.1 for Dirichlet boundary conditions is well known for more general systems from the work of Bereanu and Mawhin [12]. Therefore, we leave the proof for the reader while using directly Theorem 2.1 or topology degree theory, and we can also refer to the proof of Theorem 3.2. 


\subsection{Periodic or Neumann problems with nonlinearities}

In this section, we consider the existence of solutions of equation (1.1) with periodic boundary condition

$$
\mathrm{x}(0)-\mathrm{x}(T)=0=\mathrm{x}^{\prime}(0)-\mathrm{x}^{\prime}(T)
$$

or Neumann boundary condition

$$
\mathrm{x}^{\prime}(0)=0=\mathrm{x}^{\prime}(T)
$$

There is no universal existence for equation (1.1) with periodic boundary condition or Neumann boundary condition. The following lemma is a result of nonexistence of solutions.

Lemma 3.1 If there exists a component function $f_{i}:[0, T] \times \mathbb{R}^{3} \times \mathbb{R}^{3} \rightarrow \mathbb{R}$ such that $f_{i}$ is always positive or negative on its domain, then equation (1.1) with periodic boundary condition or Neumann boundary condition has no solution.

Proof Let $\varphi(t)$ be a solution of equation (1.1) with periodic boundary condition or Neumann boundary condition. Integrating the equation

$$
\left(\phi_{i}\left(\varphi^{\prime}(t)\right)\right)^{\prime}=\mathrm{f}_{i}\left(t, \varphi(t), \varphi^{\prime}(t)\right)
$$

with respect to $t$ on interval $[0, T]$, we have

$$
0=\int_{0}^{T} f_{i}\left(s, \varphi(s), \varphi^{\prime}(s)\right) \mathrm{d} s
$$

Since $f_{i}$ always has a positive sign or a negative sign on its domain, the integral on the right side of the equation does not vanish. Thus, it is a contradiction, which implies that equation (1.1) with periodic or Neumann boundary condition has no solution.

In the following, we will show that a general sign condition upon $f$ can guarantee the existence and multiplicity of solutions for equation (1.1) with periodic or Neumann boundary condition.

First, we consider the periodic boundary value problem

$$
\left(\phi\left(\mathrm{x}^{\prime}\right)\right)^{\prime}=\mathrm{f}\left(t, \mathrm{x}, \mathrm{x}^{\prime}\right), \quad \mathrm{x}(0)-\mathrm{x}(T)=0=\mathrm{x}^{\prime}(0)-\mathrm{x}^{\prime}(T) .
$$

A result on the existence and multiplicity of solutions is obtained in the following theorem.

Theorem 3.2 Assume that $\mathrm{f}$ is a continuous vector-valued function and there exist positive increasing sequences $\left\{\alpha_{k}^{i}\right\}$ with

$$
\alpha_{1}^{i}<\alpha_{2}^{i}<\cdots<\alpha_{n}^{i}, \quad i=1,2,3, n \geq 2, n \in \mathbb{N},
$$


and $\epsilon \in\{-1,1\}$ such that for all $t \in[0, T]$,

$$
\begin{array}{ll}
\epsilon \int_{0}^{T} f_{i}\left(s, \mathrm{x}(s), \mathrm{x}^{\prime}(s)\right) \mathrm{d} s \geq 0 & \text { if }\left|x_{i}(t)-\alpha_{k}^{i}\right|<a T,\left|x_{i}^{\prime}(t)\right|<a, \\
\epsilon \int_{0}^{T} f_{i}\left(s, \mathrm{x}(s), \mathrm{x}^{\prime}(s)\right) \mathrm{d} s \leq 0 & \text { if }\left|x_{i}(t)-\alpha_{k+1}^{i}\right|<a T,\left|x_{i}^{\prime}(t)\right|<a .
\end{array}
$$

Then periodic boundary value problem (3.2) has at least one solution. Moreover, if

$$
\sharp\left\{j \in \mathbb{Z}^{+} \mid \max _{i=1,2,3}\left(\alpha_{2 j+1}^{i}-\alpha_{2 j}^{i}\right)>2 a T\right\}=r, \quad r \in \mathbb{Z}^{+},
$$

where $\sharp(\cdot)$ denotes the number of elements in a set, then the periodic boundary value problem (3.2) has at least $r$ geometrical distinct solutions.

Proof First, assume that $\mathrm{f}$ is a continuous Lipschitz vector-valued function. Suppose that $\left(\mathrm{x}\left(t ; \mathrm{x}^{(0)}, \mathrm{y}^{(0)}\right), \mathrm{y}\left(t ; \mathrm{x}^{(0)}, \mathrm{y}^{(0)}\right)\right)$ is a solution of equation (1.1). Every zero point of the continuous mapping $\mathcal{F}=\mathcal{P}$ - id corresponds to a $T$-periodic solution of equation (1.1), where id denotes the identity map, and $\mathcal{P}$ is the Poincaré mapping. By the first equation of (2.1) we have

$$
\left|x_{i}\left(t ; \mathrm{x}^{(0)}, \mathrm{y}^{(0)}\right)-x_{i}^{(0)}\right|<a T, \quad\left|x_{i}^{\prime}\left(t ; \mathrm{x}^{(0)}, \mathrm{y}^{(0)}\right)\right|<a, \quad t \in[0, T] .
$$

Let

$$
M_{k}^{i}=\max _{t \in[0, T]}\left\{\left|f_{i}(t, \mathrm{u}, \mathrm{v})\right|: u_{j} \in\left[\alpha_{k}^{j}-2 a T, \alpha_{k}^{j}+2 a T\right], v_{j} \in[-a, a], j=1,2,3\right\} .
$$

Since $f_{i}(i=1,2,3)$ is continuous, by the boundedness of $\mathrm{x}(t)$ and $\mathrm{x}^{\prime}(t)$ on $[0, T], y_{i}^{\prime}(t)$ is also bounded on $[0, T]$. By the second equality of $(2.1)$ we can take a sufficiently large positive constant $\beta_{k}^{i}>M_{k}^{i} T$ such that

$$
\left.y_{i}\left(t ; \mathrm{x}^{(0)}, \mathrm{y}^{(0)}\right)\right|_{\mathrm{y}_{i}^{(0)}=\beta_{k}^{i}}>0
$$

and

$$
\left.y_{i}\left(t ; \mathrm{x}^{(0)}, \mathrm{y}^{(0)}\right)\right|_{\mathrm{y}_{i}^{(0)}=-\beta_{k}^{i}}<0
$$

for all $t \in[0, T]$ with $\left|x_{j}^{(0)}-\alpha_{k}^{j}\right|<a T, j=1,2,3$.

Consider the parallelotope

$$
\mathcal{D}_{k}=\left\{\left(x_{1}, x_{2}, x_{3}, y_{1}, y_{2}, y_{3}\right) \in \mathbb{R}^{6}: \alpha_{k}^{i} \leq x_{i} \leq \alpha_{k+1}^{i},-\beta_{k}^{i} \leq y_{i} \leq \beta_{k}^{i}, i=1,2,3\right\}
$$

with its boundary including 12 faces. We denote them by

$$
\begin{aligned}
& V_{-}^{i}=\left\{\left(x_{1}, x_{2}, x_{3}, y_{1}, y_{2}, y_{3}\right) \in \mathcal{D}_{k}: y_{i}=-\beta_{k}^{i}\right\}, \\
& V_{+}^{i}=\left\{\left(x_{1}, x_{2}, x_{3}, y_{1}, y_{2}, y_{3}\right) \in \mathcal{D}_{k}: y_{i}=\beta_{k}^{i}\right\},
\end{aligned}
$$




$$
\begin{aligned}
& V_{-}^{i+3}=\left\{\left(x_{1}, x_{2}, x_{3}, y_{1}, y_{2}, y_{3}\right) \in \mathcal{D}_{k}: x_{i}=\alpha_{k}^{i}\right\}, \\
& V_{+}^{i+3}=\left\{\left(x_{1}, x_{2}, x_{3}, y_{1}, y_{2}, y_{3}\right) \in \mathcal{D}_{k}: x_{i}=\alpha_{k+1}^{i}\right\}, \quad i=1,2,3,
\end{aligned}
$$

respectively. Integrating the first equality of (2.1) with respect $t$ on the interval $[0, T]$, we have

$$
\begin{aligned}
\mathcal{F}_{i}\left(V_{+}^{i}\right) & =x_{i}\left(T ; \mathrm{x}^{(0)}, \mathrm{y}^{(0)}\right)-x_{i}\left(0 ; \mathrm{x}^{(0)}, \mathrm{y}^{(0)}\right) \\
& =\left.\int_{0}^{T} \phi_{i}^{-1}\left(\mathrm{y}\left(t ; \mathrm{x}^{(0)}, \mathrm{y}^{(0)}\right)\right)\right|_{\mathrm{y}_{i}^{(0)}=\beta_{k}^{i}} \mathrm{~d} t \geq 0
\end{aligned}
$$

and

$$
\begin{aligned}
\mathcal{F}_{i}\left(V_{-}^{i}\right) & =x_{i}\left(T ; \mathrm{x}^{(0)}, \mathrm{y}^{(0)}\right)-x_{i}\left(0 ; \mathrm{x}^{(0)}, \mathrm{y}^{(0)}\right) \\
& =\left.\int_{0}^{T} \phi_{i}^{-1}\left(\mathrm{y}\left(t ; \mathrm{x}^{(0)}, \mathrm{y}^{(0)}\right)\right)\right|_{\mathrm{y}_{i}^{(0)}=-\beta_{k}^{i}} \mathrm{~d} t \leq 0
\end{aligned}
$$

for $i=1,2,3$, where the last inequality follows by the monotonicity of $\phi_{i}^{-1}$.

Integrating the second equality of (2.1) with respect $t$ on the interval $[0, T]$, by inequalities (3.3), (3.4), and (3.5) we have

$$
\begin{aligned}
\epsilon \mathcal{F}_{i+3}\left(V_{+}^{i+3}\right) & =\epsilon y_{i}\left(T ; \mathrm{x}^{(0)}, \mathrm{y}^{(0)}\right)-\epsilon y_{i}\left(0 ; \mathrm{x}^{(0)}, \mathrm{y}^{(0)}\right) \\
& =-\left.\epsilon \int_{0}^{T} f_{i}\left(t, \mathrm{x}\left(t ; \mathrm{x}^{(0)}, \mathrm{y}^{(0)}\right), \mathrm{x}^{\prime}\left(t ; \mathrm{x}^{(0)}, \mathrm{y}^{(0)}\right)\right)\right|_{x_{i}^{(0)}=\alpha_{k}^{i}} \mathrm{~d} t \leq 0
\end{aligned}
$$

and

$$
\begin{aligned}
\epsilon \mathcal{F}_{i+3}\left(V_{-}^{i+3}\right) & =\epsilon y_{i}\left(T ; \mathrm{x}^{(0)}, \mathrm{y}^{(0)}\right)-\epsilon y_{i}\left(0 ; \mathrm{x}^{(0)}, \mathrm{y}^{(0)}\right) \\
& =-\left.\epsilon \int_{0}^{T} f_{i}\left(t, \mathrm{x}\left(t ; \mathrm{x}^{(0)}, \mathrm{y}^{(0)}\right), \mathrm{x}^{\prime}\left(t ; \mathrm{x}^{(0)}, \mathrm{y}^{(0)}\right)\right)\right|_{x_{i}^{(0)}=\alpha_{k}^{i+1}} \mathrm{~d} t \geq 0
\end{aligned}
$$

for $i=1,2,3$. Thus, we have proved that

$$
\mathcal{F}_{i}\left(V_{-}^{i}\right) \mathcal{F}_{i}\left(V_{+}^{i}\right) \leq 0, \quad i=1,2, \ldots, 6
$$

Consequently, $\mathcal{F}$ satisfies the bend-twist condition on parallelotope domain $\mathcal{D}_{k}$. By Theorem 2.1, $\mathcal{F}$ has at least a zero point in domain $\mathcal{D}_{k}$. Hence, the existence of solutions is proved.

Next, we consider the multiplicity of solutions for equation (1.1) with periodic boundary condition. If, for some $i \in\{1,2,3\}$,

$$
\left|\alpha_{2 j}^{i}-\alpha_{2 j+1}^{i}\right|>2 a T
$$

then we can define two parallelotopes $\mathcal{D}_{2 j-1}$ and $\mathcal{D}_{2 j+1}$ by

$$
\begin{aligned}
& \mathcal{D}_{2 j-1}=\left\{(x, y) \in \mathbb{R}^{6}: \alpha_{2 j-1}^{i} \leq x_{i} \leq \alpha_{2 j}^{i},-\beta_{2 j-1}^{i} \leq y_{i} \leq \beta_{2 j-1}^{i}, i=1,2,3\right\}, \\
& \mathcal{D}_{2 j+1}=\left\{(x, y) \in \mathbb{R}^{6}: \alpha_{2 j+1}^{i} \leq x_{i} \leq \alpha_{2 j+2}^{i},-\beta_{2 j+1}^{i} \leq y_{i} \leq \beta_{2 j+1}^{i}, i=1,2,3\right\} .
\end{aligned}
$$


Let $\mathrm{x}^{2 j-1}$ and $\mathrm{x}^{2 j+1}$ be $T$-periodic solutions of equation (1.1) with initial values in the parallelotope domains $\mathcal{D}_{2 j-1}$ and $\mathcal{D}_{2 j+1}$, respectively. Since $x_{i}^{\prime}(t)<a$, by inequality (3.8) we have

$$
\left|x_{i}^{2 j-1}(t)-x_{i}^{2 j+1}(t)\right| \geq\left|\left(\alpha_{2 j+1}^{i}-a T\right)-\left(\alpha_{2 j}^{i}+a T\right)\right|>0
$$

for all $t \in[0, T]$. So, in this case, the two $T$-periodic solutions from two distinct parallelotope domains must be distinct.

Finally, we show that the continuity condition on $\mathrm{f}$ can take place of the Lipschitz condition in our results by the method developed by Ding et al. [32].

In fact, if $\mathrm{f}$ is a continuous function, then we can take a sequence of functions $\mathrm{f}_{l}=\mathrm{f}_{l}(t, \mathrm{x}, \mathrm{y})$ that are $T$-periodic in the $t$-variable and locally Lipschitzian in the $x, y$-variable, with $\left\{\mathrm{f}_{l}(t, \mathrm{x}, \mathrm{y})\right\}$ converging to $\mathrm{f}$ uniformly on each compact subset of $[0, T] \times \mathbb{R}^{3} \times \mathbb{R}^{3}$. The existence of such a sequence, with the $\mathrm{f}_{l}$ even smoother than required here, follows from the Weierstrass approximation theorem. Of course, we can take $\mathrm{f}_{l} \equiv \mathrm{f}(t, \mathrm{x}, \mathrm{y})$ if $f$ is locally Lipschitzian. We conclude that the equations

$$
\left(\phi\left(\mathrm{x}^{\prime}\right)\right)^{\prime}=\mathrm{f}_{l}\left(t, \mathrm{x}, \mathrm{x}^{\prime}\right)
$$

have at least one $T$-periodic solution $\mathrm{x}_{l}(t)$ with

$$
\operatorname{dist}\left(\mathrm{x}_{l}(t), \mathcal{D}\right) \leq \sqrt{3} a T, \quad \mathrm{x}_{l}^{\prime}(t)<a, \quad \forall t \in[0, T],
$$

where $\mathcal{D}$ is a uniformly bounded parallelotope domain with respect to the index $l$, and $\operatorname{dist}\left(\mathrm{x}_{l}(t), \mathcal{D}\right)$ denotes the distance from $\mathrm{x}_{l}(t)$ to $\mathcal{D}$. Now by a standard compactness argument we can find a subsequence $\left\{\mathrm{f}_{l^{\prime}}\right\}$ converging to $\mathrm{f}$ uniformly on the compact subset

$$
\mathcal{O}=\left\{(t, z) \in[0, T] \times \mathbb{R}^{6}: \operatorname{dist}(z, \mathcal{D}) \leq \sqrt{3} a T\right\}
$$

We can also find a subsequence of the $\left\{\mathrm{x}_{l^{\prime}}\right\}_{l}$ converging to $\mathrm{x}$, which is a $T$-periodic solution of equation (3.2).

We remark that conditions (3.3) and (3.4) for Neumann or periodic problems in Theorem 3.2 were first introduced by Bereanu and Mawhin, where they have established the existence result for one-dimensional nonlinear problems with singular $\phi$-Laplacian [8], Theorem 2. Moreover, the proof of Theorem 3.2 involves an idea of shooting approach to nonlinear system with singular $\phi$, and we can refer to [33].

Corollary 3.1 Let $\mathrm{p}(t, \mathrm{u}, \mathrm{v}):[0, T] \times \mathbb{R}^{3} \times \mathbb{R}^{3} \rightarrow \mathbb{R}^{3}$ and $\mathrm{f}(t, \mathrm{u}):[0, T] \times \times \mathbb{R}^{3} \rightarrow \mathbb{R}^{3}$ be continuous functions, $\mathrm{p}(t, \mathrm{u}, \mathrm{v})$ be bounded on $[0, T] \times \mathbb{R}^{3} \times B_{a}(0)$, and $\mathrm{f}$ satisfy the conditions

$$
\limsup _{u_{i} \rightarrow-\infty} f_{i}(t, \mathrm{u}) \leq \inf p_{i}(t, \mathrm{u}, \mathrm{v}), \quad \liminf _{u_{i} \rightarrow+\infty} f_{i}(t, \mathrm{u}) \geq \sup p_{i}(t, \mathrm{u}, \mathrm{v})
$$

or

$$
\liminf _{u_{i} \rightarrow-\infty} f_{i}(t, \mathrm{u}) \geq \sup p_{i}(t, \mathrm{u}, \mathrm{v}), \quad \limsup _{u_{i} \rightarrow+\infty} f_{i}(t, \mathrm{u}) \leq \inf p_{i}(t, \mathrm{u}, \mathrm{v})
$$


uniformly in $t \in[0, T]$. Then the problem

$$
\left(\phi\left(\mathrm{x}^{\prime}\right)\right)^{\prime}+\mathrm{f}(t, \mathrm{x})=\mathrm{p}\left(t, \mathrm{x}, \mathrm{x}^{\prime}\right), \quad \mathrm{x}(0)-\mathrm{x}(T)=0=\mathrm{x}^{\prime}(0)-\mathrm{x}^{\prime}(T),
$$

has at least one solution.

Proof Let (3.9) hold. Then there exist two constants $\alpha_{1}, \alpha_{2}$ with $-\alpha_{1}, \alpha_{2} \gg 1$ such that

$$
p_{i}\left(t, \mathrm{x}, \mathrm{x}^{\prime}\right)-f_{i}(t, \mathrm{x}) \geq 0 \quad \text { for }\left|x_{i}(t)-\alpha_{1}\right|<a T,\left|x_{i}^{\prime}(t)\right|<a
$$

and

$$
p_{i}\left(t, \mathrm{x}, \mathrm{x}^{\prime}\right)-f_{i}(t, \mathrm{x}) \leq 0 \quad \text { for }\left|x_{i}(t)-\alpha_{2}\right|<a T,\left|x_{i}^{\prime}(t)\right|<a,
$$

which implies that (3.3) and (3.4) are satisfied. It is a direct consequence of Theorem 3.2. The proof is similar in case (3.10) holds.

Corollary 3.2 Let $\mathrm{p}(t, \mathrm{u}, \mathrm{v}):[0, T] \times \mathbb{R}^{3} \times \mathbb{R}^{3} \rightarrow \mathbb{R}^{3}$ and $\mathrm{f}(t, \mathrm{u}):[0, T] \times \times \mathbb{R}^{3} \rightarrow \mathbb{R}^{3}$ be continuous functions, $\mathrm{p}(t, \mathrm{u}, \mathrm{v})$ be bounded on $[0, T] \times \mathbb{R}^{3} \times B_{a}(0)$, and $\mathrm{f}$ satisfy the conditions

$$
\lim _{u_{i} \rightarrow-\infty} f_{i}(t, \mathrm{u})=+\infty, \quad \lim _{u_{i} \rightarrow+\infty} \mathrm{f}(t, \mathrm{u})=-\infty
$$

or

$$
\lim _{u_{i} \rightarrow-\infty} f_{i}(t, \mathrm{u})=-\infty, \quad \lim _{u_{i} \rightarrow+\infty} f_{i}(t, \mathrm{u})=+\infty
$$

uniformly in $t \in[0, T]$. Then problem (4.10) has at least one solution.

Corollary 3.2 is easily deduced from Corollary 3.1.

Example 3.1 For any continuous and bounded $T$-periodic vector-valued function $\mathrm{p}$ and constants $\mu \neq 0$ and $\lambda>1$, the problem

$$
\begin{aligned}
& \frac{\mathrm{d}}{\mathrm{d} t}\left(\frac{\mathrm{x}^{\prime}}{\sqrt{1-\mathrm{x}^{\prime 2}}}\right)+\mu \mathrm{x}(\lambda+\sin \sqrt{|\mathrm{x}|})=\mathrm{p}\left(t, \mathrm{x}, \mathrm{x}^{\prime}\right) \\
& \mathrm{x}(0)-\mathrm{x}(T)=0=\mathrm{x}^{\prime}(0)-\mathrm{x}^{\prime}(T)
\end{aligned}
$$

has at least one solution.

These results also hold in the case of one-dimensional plane problems with singular $\phi$-Laplacian. An immediate consequence discussed by Bereanu and Mawhin ([8], Theorem 2) follows from Theorem 3.2.

Remark 3.1 It is not difficult to see that the results also hold for Neumann boundary conditions. 


\subsection{Relativistic equations of charged particles in an electromagnetic field}

Now let us return to the relativistic equations (1.3) of a charged particle in an electromagnetic field. Without loss of generality, we consider the particle of mass $m=1$. Note that we can rewrite the equations in the form

$$
\left\{\begin{array}{l}
\dot{x}_{i}=\frac{y_{i}}{\sqrt{1+\frac{|\dot{y}|^{2}}{c^{2}}}} \Leftrightarrow y_{i}=\frac{\dot{x}_{i}}{\sqrt{1-\frac{|\dot{x}|^{2}}{c^{2}}}}, \\
\dot{y}_{i}=-V_{x_{i}}(\mathbf{x})+\frac{e}{c} A_{i}(t) .
\end{array}\right.
$$

By Lemma 2.1 every solution of equations (3.12) can be uniquely defined on the interval $(-\infty,+\infty)$, provided that $V(\mathrm{x})$ is continuously differentiable. Here we require that the electric potential $V(\mathrm{x})$ satisfies the conditions

$$
\left(\mathrm{H}_{1}\right) \quad \epsilon V_{x_{i}}(\mathrm{x}) x_{i}>0 \quad \text { for }\left|x_{i}\right| \geq d,
$$

where $d$ is a constant, and $\epsilon \in\{-1,1\}$. Under hypothesis $\left(\mathrm{H}_{1}\right)$, we will investigate the periodic dynamics of system (3.12) in the generalized phase space $(x, y) \in \mathbb{R}^{3} \times \mathbb{R}^{3}$. We conclude the main result in the following:

Theorem 3.3 Assume that $\left(\mathrm{H}_{1}\right)$ holds and the electric potential $V$ is a continuous differentiable function. Then for any continuous T-periodic vector magnetic potential $\mathrm{A}(t)$ with zero mean value of every component variable, system (1.3) admits at least one T-periodic motion.

Proof The proof is similar to that of Theorem 3.2 and we only give a sketch. Without loss of generality, we assume that $\left(\mathrm{H}_{1}\right)$ holds for $\epsilon=1$. Let

$$
\vartheta_{i}=\max _{\substack{\alpha_{i} \leq x_{i} \leq \beta_{i} \\ i=1,2,3}}\left|V_{x_{i}}(x)\right| \quad \text { and } \quad\left\|A_{i}\right\|_{\infty}=\max _{t \in[0, T]}\left|A_{i}(t)\right|
$$

Consider the parallelotope

$$
\mathcal{D}=\left\{\left(x_{1}, x_{2}, x_{3}, y_{1}, y_{2}, y_{3}\right) \in \mathbb{R}^{6}: \alpha_{i+3} \leq x_{i} \leq \beta_{i+3}, \alpha_{i} \leq y_{i} \leq \beta_{i}, i=1,2,3\right\},
$$

where we fix the constants $\alpha_{j}, \beta_{j}, j=1,2, \ldots, 6$, such that

$$
\begin{aligned}
& \alpha_{i+3}<-2 c T-d, \quad \beta_{i+3}>2 c T+d, \\
& \alpha_{i}<-\vartheta_{i} T-\left\|A_{i}\right\|_{\infty}, \quad \beta_{i}>\vartheta_{i} T+\left\|A_{i}\right\|_{\infty}, \quad i=1,2,3 .
\end{aligned}
$$

Denote the faces of the boundary of the parallelotope by

$$
\begin{aligned}
& V_{-}^{i}=\left\{\left(x_{1}, x_{2}, x_{3}, y_{1}, y_{2}, y_{3}\right) \in \mathcal{D}: y_{i}=\alpha_{i}\right\}, \\
& V_{+}^{i}=\left\{\left(x_{1}, x_{2}, x_{3}, y_{1}, y_{2}, y_{3}\right) \in \mathcal{D}: y_{i}=\beta_{i}\right\}, \\
& V_{-}^{i+3}=\left\{\left(x_{1}, x_{2}, x_{3}, y_{1}, y_{2}, y_{3}\right) \in \mathcal{D}: x_{i}=\alpha_{i+3}\right\}, \\
& V_{+}^{i+3}=\left\{\left(x_{1}, x_{2}, x_{3}, y_{1}, y_{2}, y_{3}\right) \in \mathcal{D}: x_{i}=\beta_{i+3}\right\}, \quad i=1,2,3 .
\end{aligned}
$$


Note that

$$
\int_{0}^{T} A_{i}(t) \mathrm{d} t=0
$$

Defining the operator $\mathcal{F}$ as in Theorem 3.2, we can verify that

$$
\begin{aligned}
& \mathcal{F}_{i+3}\left(V_{-}^{i+3}\right) \geq 0, \quad \mathcal{F}_{i+3}\left(V_{+}^{i+3}\right) \leq 0, \\
& \mathcal{F}_{i}\left(V_{-}^{i}\right) \leq 0, \quad \mathcal{F}_{i}\left(V_{+}^{i}\right) \geq 0, \quad i=1,2,3 .
\end{aligned}
$$

Therefore, $\mathcal{F}$ satisfies the bend-twist condition, and by Theorem 2.1 we complete the proof.

Example 3.2 For any constants $m, c, \mu>0$ and $\gamma>1$ and any constant vector $F=$ $\left(F_{1}, F_{2}, F_{3}\right)$, the problem

$$
\begin{aligned}
& \frac{\mathrm{d}}{\mathrm{d} t}\left(\frac{m \dot{\mathrm{x}}}{\sqrt{1-\frac{|\dot{\mathrm{x}}|^{2}}{c^{2}}}}\right)+\mu \nabla(|\mathrm{x}|)^{\gamma}=\mathrm{F} \cos (\omega t), \\
& x(0)-x(T)=0=x^{\prime}(0)-x^{\prime}(T),
\end{aligned}
$$

has at least one solution.

\section{Generalized Liénard differential equations with periodic boundary value condition}

In this section, we investigate the generalized Liénard differential equations. The existence, multiplicity, and dependence on a parameter are considered. The results are illustrated with some examples.

\subsection{Existence of periodic solutions under a sign condition}

Now we recall the generalized Liénard differential equation

$$
\left(\phi\left(\mathrm{x}^{\prime}\right)\right)^{\prime}+\nabla \mathrm{F}(\mathrm{x}) \mathrm{x}^{\prime}+\mathrm{g}(t, \mathrm{x})=0
$$

which is equivalent to the system

$$
x_{i}^{\prime}=\phi_{i}^{-1}\left(y_{i}-F_{i}(\mathrm{x})\right), \quad y_{i}^{\prime}=-g_{i}(t, \mathrm{x}), \quad i=1,2,3,
$$

where

$$
\mathrm{F}=\left(F_{1}, F_{2}, F_{3}\right): \mathbb{R}^{3} \rightarrow \mathbb{R}^{3}
$$

is a continuously differentiable function, and

$$
\mathrm{g}=\left(g_{1}, g_{2}, g_{3}\right): \mathrm{R} \times \mathbb{R}^{3} \rightarrow \mathbb{R}^{3}
$$

is continuous and $T$-periodic with respect to the variable $t$. 
A function $\mathrm{g}(t, \mathrm{x})$ is said to satisfy the sign condition if there exist positive constants $d_{i}>0$ such that

$$
\left(\mathrm{H}_{2}\right) \quad \operatorname{sgn}\left(x_{i}\right) g_{i}(t, \mathrm{x}) \geq 0 \quad \text { or } \quad \operatorname{sgn}\left(x_{i}\right) g_{i}(t, \mathrm{x}) \leq 0
$$

for all $\left|x_{i}\right| \geq d_{i}, i=1,2,3$, and $t \in[0, T]$. If the signs of inequalities replace by ' $>$ ' and '<', we say that $\mathrm{g}(t, \mathrm{x})$ satisfies the strict sign condition. If a function $\mathrm{g}(t, \mathrm{x})$ satisfies the sign condition, then this means that $\mathrm{g}$ does not change sign on each axis direction of $\mathrm{x}$. For example, the function

$$
\mathrm{g}(t, \mathrm{x})=\frac{2+\sin t}{\sqrt{1+|\mathrm{x}|^{2}}} \mathrm{x}
$$

satisfies the sign condition for arbitrary positive constants $d_{1}, d_{2}, d_{3}$.

In what follows, we will apply Theorem 2.1 to discuss the existence of $T$-periodic solutions for equation (4.1).

Theorem 4.1 If $g$ satisfies the sign condition $\left(\mathrm{H}_{2}\right)$ and the function $\mathrm{F}$ is continuous differentiable, then for any given $T>0$, equation (4.1) has at least one T-periodic solution.

Proof Without loss of generality, we assume that

$$
g_{i}(t, \mathrm{x}) \geq 0, \quad \forall x_{i} \in\left[d_{i},+\infty\right)
$$

and

$$
g_{i}(t, \mathrm{x}) \leq 0, \quad \forall x_{i} \in\left(-\infty,-d_{i}\right]
$$

uniformly on $t \in[0, T]$. The other cases can be dealt with similarly.

Let $\left(\mathrm{x}\left(t ; \mathrm{x}^{(0)}, \mathrm{y}^{(0)}\right), \mathrm{y}\left(t ; \mathrm{x}^{(0)}, \mathrm{y}^{(0)}\right)\right)$ be a solution of equation (4.2). In the following, we will prove the existence of zero points of the mapping $\mathcal{F}=\mathcal{P}-$ id, which corresponds to a $T$-periodic solution of (4.2).

By the first equation of (4.2) we have

$$
\left|x_{i}\left(t ; \mathrm{x}^{(0)}, \mathrm{y}^{(0)}\right)-x_{i}^{(0)}\right|<a T, \quad\left|x_{i}^{\prime}\left(t ; \mathrm{x}^{(0)}, \mathrm{y}^{(0)}\right)\right|<a, \quad t \in[0, T] .
$$

Take fixed constants $\alpha^{i}>d_{i}+2 a T, i=1,2,3$. Then we get

$$
\left.g_{i}\left(t, \mathrm{x}\left(t ; \mathrm{x}^{(0)}, \mathrm{y}^{(0)}\right)\right)\right|_{\mathrm{x}_{i}^{(0)}=\alpha_{i}}>0
$$

and

$$
\left.g_{i}\left(t, \mathrm{x}\left(t ; \mathrm{x}^{(0)}, \mathrm{y}^{(0)}\right)\right)\right|_{\mathrm{x}_{i}^{(0)}=-\alpha_{i}}<0
$$

for all $t \in[0, T], i=1,2,3$.

Let

$$
M^{i}=\max \left\{\left|F_{i}(\mathrm{x})\right|: x_{i} \in\left[\alpha^{i}-2 a T, \alpha^{i}+2 a T\right] \cup\left[-\alpha^{i}-2 a T,-\alpha^{i}+2 a T\right]\right\} .
$$


Since $g_{i}(t, \mathrm{x})$ is continuous, by the boundedness of $\mathrm{x}(t)$ and $\mathrm{x}^{\prime}(t)$ on $[0, T], y_{i}^{\prime}(t)$ is also bounded on $[0, T]$. By the second equality of (4.2) we can take a sufficiently large positive constant $\beta^{i}$ such that

$$
\left.y_{i}\left(t ; \mathrm{x}^{(0)}, \mathrm{y}^{(0)}\right)\right|_{\mathrm{y}_{i}^{(0)}=\beta^{i}}-M^{i}>0
$$

and

$$
\left.y_{i}\left(t ; \mathrm{x}^{(0)}, \mathrm{y}^{(0)}\right)\right|_{y_{i}^{(0)}=-\beta^{i}}+M^{i}<0
$$

for all $t \in[0, T]$ with $\left|x_{i}^{(0)}-\alpha^{i}\right|<a T, i=1,2,3$.

Consider the parallelotope

$$
\mathcal{D}=\left\{\left(x_{1}, x_{2}, x_{3}, y_{1}, y_{2}, y_{3}\right) \in \mathbb{R}^{6}:-\alpha^{i} \leq x_{i} \leq \alpha^{i},-\beta^{i} \leq y_{i} \leq \beta^{i}, i=1,2,3\right\}
$$

with its boundary including 12 faces, denoted by

$$
\begin{aligned}
& V_{-}^{i}=\left\{\left(x_{1}, x_{2}, x_{3}, y_{1}, y_{2}, y_{3}\right) \in \mathcal{D}_{k}: y_{i}=-\beta^{i}\right\}, \\
& V_{+}^{i}=\left\{\left(x_{1}, x_{2}, x_{3}, y_{1}, y_{2}, y_{3}\right) \in \mathcal{D}_{k}: y_{i}=\beta^{i}\right\}, \\
& V_{-}^{i+3}=\left\{\left(x_{1}, x_{2}, x_{3}, y_{1}, y_{2}, y_{3}\right) \in \mathcal{D}_{k}: x_{i}=-\alpha^{i}\right\}, \\
& V_{+}^{i+3}=\left\{\left(x_{1}, x_{2}, x_{3}, y_{1}, y_{2}, y_{3}\right) \in \mathcal{D}_{k}: x_{i}=\beta^{i}\right\}, \quad i=1,2,3 .
\end{aligned}
$$

Integrating the first equality of (4.2) with respect to $t$ on the interval [0,T], by inequalities (4.6) and (4.7) we have

$$
\begin{aligned}
\mathcal{F}_{i}\left(V_{+}^{i}\right) & =x_{i}\left(T ; \mathrm{x}^{(0)}, \mathrm{y}^{(0)}\right)-x_{i}\left(0 ; \mathrm{x}^{(0)}, \mathrm{y}^{(0)}\right) \\
& =\left.\int_{0}^{T} \phi_{i}^{-1}\left(\mathrm{y}\left(t ; \mathrm{x}^{(0)}, \mathrm{y}^{(0)}\right)-F_{i}\left(\mathrm{x}\left(t ; \mathrm{x}^{(0)}, \mathrm{y}^{(0)}\right)\right)\right)\right|_{\mathrm{y}_{i}^{(0)}=\beta_{k}^{i}} \mathrm{~d} t \geq 0
\end{aligned}
$$

and

$$
\begin{aligned}
\mathcal{F}_{i}\left(V_{-}^{i}\right) & =x_{i}\left(T ; \mathrm{x}^{(0)}, \mathrm{y}^{(0)}\right)-x_{i}\left(0 ; \mathrm{x}^{(0)}, \mathrm{y}^{(0)}\right) \\
& =\left.\int_{0}^{T} \phi_{i}^{-1}\left(\mathrm{y}\left(t ; \mathrm{x}^{(0)}, \mathrm{y}^{(0)}\right)-F_{i}\left(\mathrm{x}\left(t ; \mathrm{x}^{(0)}, \mathrm{y}^{(0)}\right)\right)\right)\right|_{\mathrm{y}_{i}^{(0)}=-\beta_{k}^{i}} \mathrm{~d} t \leq 0
\end{aligned}
$$

for $i=1,2,3$, where the last inequality follows by the monotonicity of $\phi_{i}^{-1}$.

Integrating the second equality of (4.2) with respect $t$ on the interval $[0, T]$, by inequalities (4.4) and (4.5) we have

$$
\begin{aligned}
\mathcal{F}_{i+3}\left(V_{+}^{i+3}\right) & =y_{i}\left(T ; \mathrm{x}^{(0)}, \mathrm{y}^{(0)}\right)-y_{i}\left(0 ; \mathrm{x}^{(0)}, \mathrm{y}^{(0)}\right) \\
& =-\left.\int_{0}^{T} g_{i}\left(t, \mathrm{x}\left(t ; \mathrm{x}^{(0)}, \mathrm{y}^{(0)}\right)\right)\right|_{x_{i}^{(0)}=\alpha_{k}^{i}} \mathrm{~d} t \leq 0
\end{aligned}
$$


and

$$
\begin{aligned}
\mathcal{F}_{i+3}\left(V_{-}^{i+3}\right) & =y_{i}\left(T ; \mathrm{x}^{(0)}, \mathrm{y}^{(0)}\right)-y_{i}\left(0 ; \mathrm{x}^{(0)}, \mathrm{y}^{(0)}\right) \\
& =-\left.\int_{0}^{T} g_{i}\left(t, \mathrm{x}\left(t ; \mathrm{x}^{(0)}, \mathrm{y}^{(0)}\right)\right)\right|_{x_{i}^{(0)}=-\alpha^{i}} \mathrm{~d} t \geq 0
\end{aligned}
$$

for $i=1,2,3$. Thus, we have proved that

$$
\mathcal{F}_{i}\left(V_{-}^{i}\right) \mathcal{F}_{i}\left(V_{+}^{i}\right)<0, \quad i=1,2, \ldots, 6
$$

Consequently, $\mathcal{F}$ satisfies the bend-twist condition on the parallelotope domain $\mathcal{D}$. By Theorem 2.1, $\mathcal{F}$ has at least a zero point in $\mathcal{D}$. Hence, the existence of solutions is proved.

It is interesting to note that no assumption on the friction term $F(x)$ is required. Some direct consequences follow from Theorem 2.1.

Corollary 4.1 Assume that a continuous function $\mathrm{g}: \mathbb{R} \times \mathbb{R}^{3} \rightarrow \mathbb{R}^{3}$ satisfies

$$
\lim _{x_{i} \rightarrow-\infty} g_{i}(t, \mathrm{x})=+\infty, \quad \lim _{x_{i} \rightarrow+\infty} g_{i}(t, \mathrm{x})=-\infty, \quad i=1,2,3
$$

or

$$
\lim _{x_{i} \rightarrow-\infty} g_{i}(t, \mathrm{x})=-\infty, \quad \lim _{x_{i} \rightarrow+\infty} g_{i}(t, \mathrm{x})=+\infty, \quad i=1,2,3
$$

Then for any continuous function $\mathrm{F}(\mathrm{x})$, equation (4.1) has at least one T-periodic solution.

Proof The limits $\lim _{x_{i} \rightarrow \pm \infty} g_{i}(t, \mathrm{x})= \pm \infty\left(\right.$ or $\left.\lim _{x_{i} \rightarrow \pm \infty} g_{i}(t, \mathrm{x})=\mp \infty\right)$ imply that there exist $d_{i}>0(i=1,2,3)$ such that $x_{i} g_{i}(t, \mathrm{x})>0\left(\right.$ or $\left.x_{i} g_{i}(t, \mathrm{x})<0\right)$ for all $t \in \mathbb{R}$ and $\left|x_{i}\right|>d_{i}$. Therefore, sign condition $\left(\mathrm{H}_{2}\right)$ holds. By applying Theorem 4.1 we end the proof.

Example 4.1 If $e(t) \in C_{T} \times C_{T} \times C_{T}, c \in \mathbb{R} \backslash 0, d \in \mathbb{R}, q \geq 0$, and $p>1$, then the problem

$$
\frac{\mathrm{d}}{\mathrm{d} t}\left(\frac{\mathrm{x}^{\prime}}{\sqrt{1-\left|\mathrm{x}^{\prime}\right|^{2}}}\right)+d|\mathrm{x}|^{q} \mathrm{x}^{\prime}+c|\mathrm{x}|^{p-1} \mathrm{x}=e(t)
$$

has at least one $T$-periodic solution.

Notice that when $i=1$, the equation drops into a one-dimensional system. The results discussed before also hold. A direct consequence discussed in [22] is the following example.

Example 4.2 The equation

$$
\frac{\mathrm{d}}{\mathrm{d} t}\left(\frac{m x^{\prime}}{\sqrt{1-\frac{x^{\prime 2}}{c^{2}}}}\right)+\frac{1}{2} x^{3}-\frac{1}{2} x=-F_{0} \cos \omega t
$$

has at least one $\frac{2 \pi}{\omega}$-periodic solution. 


\subsection{Existence and multiplicity of periodic solutions without sign condition}

In most cases, the function $g$ does not satisfy sign condition $\left(\mathrm{H}_{1}\right)$. It is obvious that Theorem 4.1 cannot be applied to the relativistic pendulum-type equations since the potentials of pendulum-type equations are fluctuating and oscillating. But in this circumstance, the pendulum-type equations satisfy some sign property locally.

Let us consider the equation

$$
\left(\phi\left(\mathrm{x}^{\prime}\right)\right)^{\prime}+\nabla \mathrm{F}(\mathrm{x}) \mathrm{x}^{\prime}+\mathrm{g}(t, \mathrm{x})=0
$$

where

$$
\mathrm{F}=\left(F_{1}, F_{2}, F_{3}\right): \mathbb{R}^{3} \rightarrow \mathbb{R}^{3}
$$

is a continuously differentiable function, and

$$
\mathrm{g}=\left(g_{1}, g_{2}, g_{3}\right): \mathbb{R} \times \mathbb{R}^{3} \rightarrow \mathbb{R}^{3}
$$

is a continuous function with infinitely many zero points with respect to the variable $\mathrm{x}$ and is $T$-periodic with respect to the variable $t$.

Rewrite equation (4.8) in the equivalent form

$$
x_{i}^{\prime}=\phi_{i}^{-1}\left(y_{i}-F_{i}(\mathrm{x})\right), \quad y_{i}^{\prime}=-g_{i}(t, \mathrm{x}), \quad i=1,2,3 .
$$

Now we conclude by the following result on the existence and multiplicity of $T$-periodic solutions.

Theorem 4.2 Assume that there exist positive increasing sequences $\left\{\alpha_{k}^{i}\right\}$ with

$$
\alpha_{1}^{i}<\alpha_{2}^{i}<\cdots<\alpha_{n}^{i}, \quad i=1,2,3, n \geq 2, n \in \mathbb{N}
$$

and $\epsilon \in\{-1,1\}$ such that for all $t \in[0, T]$,

$$
\begin{array}{ll}
\epsilon \int_{0}^{T} g_{i}(s, \mathrm{x}(s)) \mathrm{d} s \geq 0 & \text { if }\left|x_{i}(t)-\alpha_{k}^{i}\right|<a T \\
\epsilon \int_{0}^{T} g_{i}(s, \mathrm{x}(s)) \mathrm{d} s \leq 0 & \text { if }\left|x_{i}(t)-\alpha_{k+1}^{i}\right|<a T .
\end{array}
$$

Then equation (4.8) has at least one T-periodic solution. Moreover, if

$$
\sharp\left\{j \in \mathbb{Z}^{+} \mid \max _{i=1,2,3}\left(\alpha_{2 j+1}^{i}-\alpha_{2 j}^{i}\right)>2 a T\right\}=r, \quad r \in \mathbb{Z}^{+},
$$

where $\sharp(\cdot)$ denotes the number of elements in a set, then equation (4.8) has at least $r$ geometric distinct T-periodic solutions.

The proof of Theorem 4.2 is a simple adaptation of that one of Theorem 3.2, and we do not repeat it here. 
Obviously, the conclusion of Theorem 4.2 also holds for a one-dimensional system. In the following, we shall perform an application of Theorem 4.2 to the following onedimensional relativistic pendulum-type equation with parameter $s$ :

$$
\left(\phi\left(x^{\prime}\right)\right)^{\prime}+f(x) x^{\prime}+g(x)=p(t)+s,
$$

where

$$
\phi:(-a, a) \rightarrow \mathbb{R} \quad(0<a<+\infty)
$$

is a monotonous (without loss of generality, assume that it is increasing) homeomorphism such that $\phi(0)=0, f$ and $g$ are continuous functions, and $p$ is continuous and $T$-periodic with zero mean value. Rewrite equation (4.12) in the equivalent form

$$
x^{\prime}=\phi_{i}^{-1}(y-F(x)), \quad y^{\prime}=-g(x)+p(t)+s,
$$

where

$$
F(x)=\int_{0}^{x} f(\zeta) \mathrm{d} \zeta
$$

is the primitive function of $f$.

Without loss of generality, we assume that there exist an increasing sequence $\left\{\xi_{k}\right\}, k=$ $1,2, \ldots, n, n \in \mathbb{N}$, positive sequences $\left\{b_{k}\right\}$ and $\left\{c_{k}\right\}$, and $\epsilon \in\{-1,1\}$ such that

$$
\left(\mathrm{H}_{3}\right) \quad \epsilon \operatorname{sgn}\left(x-\xi_{k}\right) g(x) \geq 0, \quad \forall x \in\left[-b_{k}+\xi_{k}, \xi_{k}\right] \cup\left[\xi_{k}, c_{k}+\xi_{k}\right]
$$

Let

$$
g_{\max }=\max \left\{g(x): x \in\left[-b_{k}+\xi_{k}, \xi_{k}\right]\right\}
$$

and

$$
g_{\min }=\min \left\{g(x): x \in\left[\xi_{k}, c_{k}+\xi_{k}\right]\right\}
$$

Theorem 4.3 Assume that $\left(\mathrm{H}_{3}\right)$ holds and

$$
T \leq \min \left\{\frac{b_{k}}{2 a}, \frac{c_{k}}{2 a}\right\}
$$

Then for any $p(t) \in \widetilde{C}_{T}$ and any continuous function $f(x)$, equation (4.12) has at least one $T$-periodic solution if s satisfies $g_{\max }<s \leq 0$ or $0 \leq s<g_{\min }$.

Moreover, if $\xi_{k+1}-\xi_{k} \geq\left(b_{k+1}+c_{k}\right) / 2+2 a T, k=1,2, \ldots, n-1$, then equation (4.12) has at least $n$ geometric distinct $T$-periodic solutions $x_{k}(t)$ such that $x_{k+1}(t)-x_{k}(t)>0, k=$ $1,2, \ldots, n-1$.

Proof Assume that

$$
\operatorname{sgn}\left(x-\xi_{k}\right) g(x) \geq 0, \quad \forall x \in\left[-b_{k}+\xi_{k}, \xi_{k}\right] \cup\left[\xi_{k}, c_{k}+\xi_{k}\right]
$$


Let

$$
\alpha_{k}=-\frac{b_{k}}{2}+\xi_{k}, \quad \tilde{\alpha}_{k+1}=\frac{c_{k}}{2}+\xi_{k} .
$$

By assumption (4.14) we have

$$
g(x(t)) \leq 0 \quad \text { if }\left|x(t)-\alpha_{k}\right| \leq a T
$$

and

$$
g(x(t)) \geq 0 \quad \text { if }\left|x(t)-\tilde{\alpha}_{k+1}\right| \leq a T .
$$

When $g_{\max }<s \leq 0$, we have

$$
\begin{aligned}
& -\int_{0}^{T}[g(x(s))-s] \mathrm{d} s+\int_{0}^{T} p(s) \mathrm{d} s \\
& \quad \geq-\int_{0}^{T}\left[g_{\max }-s\right] \mathrm{d} s \geq 0 \quad \text { if }\left|x(t)-\alpha_{k}\right|<a T
\end{aligned}
$$

and

$$
-\int_{0}^{T}[g(x(s))-s] \mathrm{d} s+\int_{0}^{T} p(s) \mathrm{d} s \leq 0 \quad \text { if }\left|x(t)-\tilde{\alpha}_{k+1}\right|<a T .
$$

Then conditions (4.10) and (4.11) are satisfied. By Theorem 4.2 we know that equation (4.12) has at least one $T$-periodic solution. The proof of the case $0 \leq s<g_{\min }$ can be dealt with similarly.

In the following, we will give a concise proof for the multiplicity of $T$-periodic solutions independent of Theorem 4.2. Notice that the $x$-component of the initial value $\left(x_{k}(0), y_{k}(0)\right)$ of periodic solution $\left(x_{k}(t), y_{k}(t)\right)$ belongs to $\left[\alpha_{k}, \tilde{\alpha}_{k+1}\right]$ by the bend-twist theorem. On the other hand, since $\left|x_{k}^{\prime}(t)\right|<a$ for $t \in[0, T]$, we have $x_{k}(t)<x_{k}(0)+a T \leq \tilde{\alpha}_{k+1}+a T$ and $x_{k}(t)>x_{k}(0)-a T \geq \alpha_{k}-a T$. Therefore, when $\xi_{k+1}-\xi_{k}>\left(b_{k+1}+c_{k}\right) / 2+2 a T, k=1,2, \ldots, n-1$, we have

$$
\begin{aligned}
x_{k+1}(t)-x_{k}(t) & >\left(\alpha_{k+1}-a T\right)-\left(\tilde{\alpha}_{k+1}+a T\right) \\
& =\xi_{k+1}-\xi_{k}-\left(b_{k+1}+c_{k}\right) / 2-2 a T \geq 0 .
\end{aligned}
$$

Thus, we end the proof.

The existence of $T$-periodic solutions of the particular relativistic pendulum equation

$$
\left(\frac{x^{\prime}}{\sqrt{1-\frac{x^{\prime 2}}{c^{2}}}}\right)^{\prime}+k x^{\prime}+a \sin x=p(t)
$$

was first considered by Torres [23]. Applying Theorem 4.3 to equation (4.15) we have the following result. 
Corollary 4.2 For any values $a, k$ and for any $p(t) \in \widetilde{C}_{T}$, equation (4.15) has at least one $T$-periodic solution, provided that $2 c T<\pi$.

Proof Equation (4.15) is equivalent to

$$
\left(\frac{x^{\prime}}{\sqrt{c^{2}-x^{\prime 2}}}\right)^{\prime}+\frac{k}{c} x^{\prime}+\frac{a}{c} \sin x=\frac{1}{c} p(t) .
$$

Take $b_{k}=\pi=c_{k}$ and $\xi_{k}=k \pi$. Obviously, condition $\left(\mathrm{H}_{3}\right)$ holds, and

$$
T \leq \frac{\pi}{2 c}=\min \left\{\frac{b_{k}}{2 a}, \frac{c_{k}}{2 a}\right\} .
$$

Applying Theorem 4.2, we conclude.

Obviously, we have generalized the result [23], Theorem 3. It is worth mentioning that for the particular $\sin x$ in equation (4.15), Torres has improved his method and obtained a better result [24], Corollary 3. However, for general $g$, his method strictly depends on the differentiability of the function $g$; see [24], Theorem 4 . Note that our results do not need any information on the differentiability of $g$.

Remark 4.1 We can see that if $x(t)$ is a $T$-periodic solution of equation (4.15), then $x(t)+$ $2 k \pi, k \in \mathbb{Z}$, also is a $T$-periodic solution. In fact, there exists a positive increasing sequence $\left\{\xi_{k}=k \pi\right\}_{k=1}^{+\infty}$ that satisfies condition $\left(\mathrm{H}_{3}\right)$ of Theorem 4.3.

Furthermore, it is interesting to note that no assumption on the friction coefficient $f(x)$ is required. So we obtain the following simple result.

Corollary 4.3 For any continuous function $f(x)$ and for any $p(t) \in \widetilde{C}_{T}$, the equation

$$
\left(\phi\left(x^{\prime}\right)\right)^{\prime}+f(x) x^{\prime}+a \sin x=p(t)
$$

has at least one T-periodic solution, provided that $2 a T<\pi$.

Example 4.3 For any $p(t) \in \widetilde{C}_{T}, b \in \mathbb{R}$, and $q>1$, the problem

$$
\begin{aligned}
& \left(\frac{x^{\prime}}{\sqrt{1-x^{\prime 2}}}\right)^{\prime}+|x|^{q+1} x-b^{2}|x|^{q-1} x=p(t), \\
& x(0)-x(T)=0=x^{\prime}(0)-x^{\prime}(T),
\end{aligned}
$$

has at least one solution. Moreover, if $T<|b| / 4$, then the problem has at least three solutions.

In case $g(x)$ does not satisfy $\left(\mathrm{H}_{3}\right)$, also $T$-periodic solutions of equation (4.12) can exist. In the following, we will consider the existence of $T$-periodic solutions with some other conditions on $g$.

Theorem 4.4 Assume that $g(x)$ satisfies

$$
g(x)>0 \quad \text { (respectively, } g(x)<0) \quad \text { for all } x \in \mathbb{R}
$$


and

$$
\lim _{x \rightarrow \pm \infty} g(x)=0
$$

Let

$$
\begin{aligned}
& s^{*}=\max _{x \in(-\infty,+\infty)} g(x), \quad s_{*}=\min _{x \in[-2 a T, 2 a T]} g(x) \\
& \text { (respectively, } \left.s^{\dagger}=\min _{x \in(-\infty,+\infty)} g(x), s_{\dagger}=\max _{x \in[-2 a T, 2 a T]} g(x)\right) .
\end{aligned}
$$

For any continuous function $f(x)$ and $p(t) \in \widetilde{C}_{T}$, there exist two positive constants $s_{*}<s^{*}$ such that if $s \notin\left(0, s^{*}\right]$ (respectively, $s \notin\left[s^{\dagger}, 0\right)$ ), then equation (4.12) has no T-periodic solution, and if $s \in\left(0, s_{*}\right]$ (respectively, $s \in\left[s_{\dagger}, 0\right)$ ), then equation (4.12) has at least two distinct T-periodic solutions.

Proof We only consider the case of $g(x)>0$, and the other case can be done similarly. Equation (4.12) is equivalent to the system

$$
\left\{\begin{array}{l}
x^{\prime}=\phi^{-1}(y-F(x)) \\
y^{\prime}=-g(x)+p(t)+s
\end{array}\right.
$$

For any $s \in\left(s^{*},+\infty\right)$, using the second equality of (4.19), we have

$$
y(T)-y(0)=\int_{0}^{T}[-g(x(\tau))+s] \mathrm{d} \tau+\int_{0}^{T} p(\tau) \mathrm{d} \tau=\int_{0}^{T}[-g(x(\tau))+s] \mathrm{d} \tau>0,
$$

which implies that equation (4.12) has no $T$-periodic solution. Moreover, for any $s \in$ $(-\infty, 0]$, we have

$$
y(T)-y(0)=\int_{0}^{T}[-g(x(\tau))+s] \mathrm{d} \tau+\int_{0}^{T} p(\tau) \mathrm{d} \tau=\int_{0}^{T}[-g(x(\tau))+s] \mathrm{d} \tau<0,
$$

which also implies that equation (4.12) has no $T$-periodic solution.

From the limits $\lim _{x \rightarrow \pm \infty} g(x)=0$ we know that for any $s \in\left(0, s_{*}\right]$, there exists a constant $X_{0}>0$ such that

$$
0<g(x)<s, \quad \forall|x|>X_{0}
$$

Denote

$$
\begin{aligned}
& g_{0}=\max _{x \in\left[0, X_{0}+2 a T\right]}|g(x)|, \quad F_{0}=\max _{x \in\left[0, X_{0}+2 a T\right]}|F(x)|, \\
& \gamma=F_{0}+\left(g_{0}+\|p\|_{\infty}+s\right) T+1 .
\end{aligned}
$$

Consider the rectangle

$$
\mathcal{D}=\left\{(x, y) \in \mathbb{R}^{2}: a T \leq x \leq X_{0}+a T,-\gamma \leq y \leq \gamma\right\},
$$


where the boundary is constituted by four segments

$$
\begin{aligned}
& V_{-}^{1}=\{(x, y) \in \mathcal{D} \mid x=a T\}, \\
& V_{+}^{1}=\left\{(x, y) \in \mathcal{D} \mid x=X_{0}+a T\right\}, \\
& V_{-}^{2}=\{(x, y) \in \mathcal{D} \mid y=-\gamma\}, \\
& V_{+}^{2}=\{(x, y) \in \mathcal{D} \mid y=\gamma\} .
\end{aligned}
$$

Let $\left(x\left(t ; x_{0}, y_{0}\right), y\left(t ; x_{0}, y_{0}\right)\right)$ be a solution of (4.19) with initial value $\left(x_{0}, y_{0}\right)$. Define the continuous mapping $\mathcal{F}: \mathbb{R}^{2} \rightarrow \mathbb{R}^{2}$ by $\mathcal{F}=\left(\mathcal{F}_{1}, \mathcal{F}_{2}\right)=\mathcal{P}-$ id, where $\mathcal{P}$ denotes the Poincaré mapping associated to system (4.19).

When $\left(x_{0}, y_{0}\right) \in V_{-}^{1}$, using the inequality $\left|x^{\prime}(t)\right|<a$, from the first equality in (4.19) we know that

$$
0<x(t)=x_{0}+\int_{0}^{t} x^{\prime}(\tau) \mathrm{d} \tau<2 a T, \quad \forall t \in[0, T] .
$$

For any $s \in\left(0, s_{*}\right]$, using the definition of $s_{*}$, we know that $g(x(t)) \geq s$ for all $t \in[0, T]$. Then it follows that

$$
\begin{aligned}
y(T)-y(0) & =\int_{0}^{T}[-g(x(\tau))+s] \mathrm{d} \tau+\int_{0}^{T} p(\tau) \mathrm{d} \tau \\
& =\int_{0}^{T}[-g(x(\tau))+s] \mathrm{d} \tau \leq 0 .
\end{aligned}
$$

When $\left(x_{0}, y_{0}\right) \in V_{+}^{1}$, using the inequality $\left|x^{\prime}(t)\right|<a$, from the first equality in (4.19) we know that

$$
X_{0}<x(t)=x_{0}+\int_{0}^{t} x^{\prime}(\tau) \mathrm{d} \tau<X_{0}+2 a T, \quad \forall t \in[0, T] .
$$

For any $s \in\left(0, s_{*}\right]$, using (4.20), we know that $g(x(t)) \leq s$ for all $t \in[0, T]$. Then it follows that

$$
\begin{aligned}
y(T)-y(0) & =\int_{0}^{T}[-g(x(\tau))+s] \mathrm{d} \tau+\int_{0}^{T} p(\tau) \mathrm{d} \tau \\
& =\int_{0}^{T}[-g(x(\tau))+s] \mathrm{d} \tau \geq 0 .
\end{aligned}
$$

Therefore, using (4.21) and (4.22), we have

$$
\mathcal{F}_{2}\left(V_{-}^{1}\right) \mathcal{F}_{2}\left(V_{+}^{1}\right) \leq 0
$$

On the other hand, when $\left(x_{0}, y_{0}\right) \in V_{-}^{2}$, using the inequality $\left|x^{\prime}(t)\right|<a$, from the first equality in (4.19) we know that $0<x(t)<X_{0}+2 a T$ for all $t \in[0, T]$. For any $s \in\left(0, s_{*}\right]$, using the second equality of (4.19) and the definition of $\gamma$, we know that

$$
\begin{aligned}
y(t) & =y(0)+\int_{0}^{t}[-g(x(\tau))+p(\tau)+s] \mathrm{d} \tau \\
& \leq-\gamma+\left(g_{0}+\|p\|_{\infty}+s\right) T=-F_{0}-1, \quad \forall t \in[0, T],
\end{aligned}
$$


which yields that $y(t)-F(x(t)) \leq-F_{0}-1-F(x(t))<0$ for all $t \in[0, T]$ by the definition of $F_{0}$. Since $\phi^{-1}: \mathbb{R} \rightarrow(-a, a)$ is a increasing homeomorphism such that $\phi^{-1}(0)=0$, we have $x^{\prime}(t)=\phi^{-1}(y-F(x))<0$ for all $t \in[0, T]$. Then it follows that $x(T)-x(0)<0$. When $\left(x_{0}, y_{0}\right) \in V_{+}^{2}$, we also know that $0<x(t)<X_{0}+2 a T$ for all $t \in[0, T]$. For any $s \in\left(0, s_{*}\right]$, we have

$$
\begin{aligned}
y(t) & =y(0)+\int_{0}^{t}[-g(x(\tau))+p(\tau)+s] \mathrm{d} \tau \\
& \geq \gamma-\left(g_{0}+\|p\|_{\infty}+s\right) T=F_{0}+1, \quad \forall t \in[0, T],
\end{aligned}
$$

which yields that $y(t)-F(x(t)) \geq F_{0}+1-F(x(t))>0$ for all $t \in[0, T]$. Then it follows that $x(T)-x(0)>0$. Therefore, we have

$$
\mathcal{F}_{1}\left(V_{-}^{2}\right) \mathcal{F}_{1}\left(V_{+}^{2}\right) \leq 0
$$

Now we have verified that $\mathcal{F}$ satisfies the bend-twist condition on $\mathcal{D}$. By Theorem 2.1 there exists at least one point $\left(x_{1}, y_{1}\right) \in \mathcal{D}$ such that $\mathcal{F}\left(x_{1}, y_{1}\right)=0$, which corresponds to a fixed point of the Poincaré mapping.

Similarly, we consider the rectangle

$$
\mathcal{D}^{\prime}=\left\{(x, y) \in \mathbb{R}^{2}:-X_{0}-a T \leq x \leq-a T,-\gamma \leq y \leq \gamma\right\} .
$$

By the same arguments we can verify that $\mathcal{F}$ satisfies the bend-twist condition on $\partial \mathcal{D}^{\prime}$ and obtain another fixed point of the Poincaré mapping in $\mathcal{D}^{\prime}$. Since $\mathcal{D}$ and $\mathcal{D}^{\prime}$ are disjoint, we obtain two distinct fixed points, which correspond to two distinct $T$-periodic solutions of equation (4.12).

Similarly, we get the following theorem.

Theorem 4.5 Assume that $g(x)$ satisfies

$$
\left.\lim _{x \rightarrow \pm \infty} g(x)=+\infty \quad \text { (respectively, } \lim _{x \rightarrow \pm \infty} g(x)=-\infty\right) .
$$

Let

$$
\begin{aligned}
& s_{*}=\min _{x \in(-\infty,+\infty)} g(x), \quad s^{*}=\max _{x \in[-2 a T, 2 a T]} g(x) \\
& \left(\text { respectively, } s_{\dagger}=\max _{x \in(-\infty,+\infty)} g(x), s^{\dagger}=\min _{x \in[-2 a T, 2 a T]} g(x)\right) .
\end{aligned}
$$

For any continuous function $f(x)$ and $p(t) \in \widetilde{C}_{T}$, if $s \in\left(-\infty, s_{*}\right)$ (respectively, $\left(s_{\dagger},+\infty\right)$ ), then equation (4.12) has no T-periodic solution, and if $\in\left[s^{*},+\infty\right)$ (respectively, $\left.\left(-\infty, s^{\dagger}\right]\right)$, then equation (4.12) has at least distinct two T-periodic solutions.

Proof We only consider the case of $\lim _{x \rightarrow \pm \infty} g(x)=+\infty$, and the other case can be done similarly. Equation (4.12) is equivalent to the system

$$
\left\{\begin{array}{l}
x^{\prime}=\phi^{-1}(y-F(x)), \\
y^{\prime}=-g(x)+p(t)+s .
\end{array}\right.
$$


For any $s \in\left(-\infty, s_{*}\right)$, using the second equality of (4.23), we have

$$
\begin{aligned}
y(T)-y(0) & =\int_{0}^{T}[-g(x(\tau))+s] \mathrm{d} \tau+\int_{0}^{T} p(\tau) \mathrm{d} \tau \\
& =\int_{0}^{T}[-g(x(\tau))+s] \mathrm{d} \tau<0,
\end{aligned}
$$

which implies that equation (4.12) has no $T$-periodic solution.

From the limits $\lim _{x \rightarrow \pm \infty} g(x)=+\infty$ we know that for any $s \in\left[s^{*},+\infty\right)$, there exists a constant $X_{0}>0$ such that

$$
g(x)>s, \quad \forall|x|>X_{0}
$$

Denote

$$
\begin{aligned}
& g_{0}=\max _{x \in\left[0, X_{0}+2 a T\right]}|g(x)|, \quad F_{0}=\max _{x \in\left[0, X_{0}+2 a T\right]}|F(x)|, \\
& \gamma=F_{0}+\left(g_{0}+\|p\|_{\infty}+s\right) T+1 .
\end{aligned}
$$

Let us consider the rectangle

$$
\mathcal{D}=\left\{(x, y) \in \mathbb{R}^{2}: a T \leq x \leq X_{0}+a T,-\gamma \leq y \leq \gamma\right\}
$$

where the boundary is constituted by four segments

$$
\begin{aligned}
& V_{-}^{1}=\{(x, y) \in \mathcal{D} \mid x=a T\}, \\
& V_{+}^{1}=\left\{(x, y) \in \mathcal{D} \mid x=X_{0}+a T\right\}, \\
& V_{-}^{2}=\{(x, y) \in \mathcal{D} \mid y=-\gamma\}, \\
& V_{+}^{2}=\{(x, y) \in \mathcal{D} \mid y=\gamma\} .
\end{aligned}
$$

Let $\left(x\left(t ; x_{0}, y_{0}\right), y\left(t ; x_{0}, y_{0}\right)\right)$ be a solution of (4.23) with initial value $\left(x_{0}, y_{0}\right)$. Define the continuous mapping $\mathcal{F}: \mathbb{R}^{2} \rightarrow \mathbb{R}^{2}$ by $\mathcal{F}=\left(\mathcal{F}_{1}, \mathcal{F}_{2}\right)=\mathcal{P}$ - id.

When $\left(x_{0}, y_{0}\right) \in V_{-}^{1}$, using the inequality $\left|x^{\prime}(t)\right|<a$, from the first equality in (4.23) we know that

$$
0<x(t)=x_{0}+\int_{0}^{t} x^{\prime}(\tau) \mathrm{d} \tau<2 a T, \quad \forall t \in[0, T]
$$

For any $s \in\left[s^{*},+\infty\right)$, using the definition of $s^{*}$, we know that $g(x(t)) \leq s$ for all $t \in[0, T]$. Then it follows that

$$
\begin{aligned}
y(T)-y(0) & =\int_{0}^{T}[-g(x(\tau))+s] \mathrm{d} \tau+\int_{0}^{T} p(\tau) \mathrm{d} \tau \\
& =\int_{0}^{T}[-g(x(\tau))+s] \mathrm{d} \tau \geq 0 .
\end{aligned}
$$


When $\left(x_{0}, y_{0}\right) \in V_{+}^{1}$, using the inequality $\left|x^{\prime}(t)\right|<a$, from the first equality in (4.23) we know that

$$
X_{0}<x(t)=x_{0}+\int_{0}^{t} x^{\prime}(\tau) \mathrm{d} \tau<X_{0}+2 a T, \quad \forall t \in[0, T]
$$

For any $s \in\left[s^{*},+\infty\right)$, using $(4.24)$, we know that $g(x(t)) \geq s$ for all $t \in[0, T]$. Then it follows that

$$
\begin{aligned}
y(T)-y(0) & =\int_{0}^{T}[-g(x(\tau))+s] \mathrm{d} \tau+\int_{0}^{T} p(\tau) \mathrm{d} \tau \\
& =\int_{0}^{T}[-g(x(\tau))+s] \mathrm{d} \tau \leq 0 .
\end{aligned}
$$

Therefore, using (4.25) and (4.26), we have

$$
\mathcal{F}_{2}\left(V_{-}^{1}\right) \mathcal{F}_{2}\left(V_{+}^{1}\right) \leq 0
$$

On the other hand, when $\left(x_{0}, y_{0}\right) \in V_{-}^{2}$, using the inequality $\left|x^{\prime}(t)\right|<a$, from the first equality in (4.23) we know that $0<x(t)<X_{0}+2 a T$ for all $t \in[0, T]$. For any $s \in\left[s^{*},+\infty\right)$, using the second equality of (4.23) and the definition of $\gamma$, we know that

$$
\begin{aligned}
y(t) & =y(0)+\int_{0}^{t}[-g(x(\tau))+p(\tau)+s] \mathrm{d} \tau \\
& \leq-\gamma+\left(g_{0}+\|p\|_{\infty}+s\right) T=-F_{0}-1, \quad \forall t \in[0, T],
\end{aligned}
$$

which yields that $y(t)-F(x(t)) \leq-F_{0}-1-F(x(t))<0$ for all $t \in[0, T]$ by the definition of $F_{0}$. Since $\phi^{-1}: \mathbb{R} \rightarrow(-a, a)$ is a increasing homeomorphism such that $\phi^{-1}(0)=0$, we have $x^{\prime}(t)=\phi^{-1}(y-F(x))<0$ for all $t \in[0, T]$. Then it follows that $x(T)-x(0)<0$. When $\left(x_{0}, y_{0}\right) \in V_{+}^{2}$, we also know that $0<x(t)<X_{0}+2 a T$ for all $t \in[0, T]$. For any $s \in\left[s^{*},+\infty\right)$, we have

$$
\begin{aligned}
y(t) & =y(0)+\int_{0}^{t}[-g(x(\tau))+p(\tau)+s] \mathrm{d} \tau \\
& \geq \gamma-\left(g_{0}+\|p\|_{\infty}+s\right) T=F_{0}+1, \quad \forall t \in[0, T],
\end{aligned}
$$

which yields that $y(t)-F(x(t)) \geq F_{0}+1-F(x(t))>0$ for all $t \in[0, T]$. Then it follows that $x(T)-x(0)>0$. Therefore, we have

$$
\mathcal{F}_{1}\left(V_{-}^{2}\right) \mathcal{F}_{1}\left(V_{+}^{2}\right) \leq 0
$$

Now we have verified that $\mathcal{F}$ satisfies the bend-twist condition on $\mathcal{D}$. By Theorem 2.1 there exists at least one point $\left(x_{1}, y_{1}\right) \in \mathcal{D}$ such that $\mathcal{F}\left(x_{1}, y_{1}\right)=0$, which is corresponding to a fixed point of the Poincaré mapping.

Similarly, consider the rectangle

$$
\mathcal{D}^{\prime}=\left\{(x, y) \in \mathbb{R}^{2}:-X_{0}-a T \leq x \leq-a T,-\gamma \leq y \leq \gamma\right\} .
$$


By the same arguments we can verify that $\mathcal{F}$ satisfies the bend-twist condition on $\partial \mathcal{D}^{\prime}$ and obtain another fixed point of the Poincaré mapping in $\mathcal{D}^{\prime}$. Since $\mathcal{D}$ and $\mathcal{D}^{\prime}$ are disjoint, we obtain two distinct fixed points, which are corresponding to two distinct $T$-periodic solutions of equation (4.12).

Theorem 4.6 Assume that $g:(-\infty,+\infty) \rightarrow(0,+\infty)$ is continuous and satisfies

$$
\lim _{u \rightarrow-\infty} g(u)=+\infty, \quad \lim _{u \rightarrow+\infty} g(u)=0
$$

or

$$
\lim _{u \rightarrow-\infty} g(u)=0, \quad \lim _{u \rightarrow+\infty} g(u)=+\infty
$$

For any $p(t) \in \widetilde{C}_{T}$, if $s>0$, then equation (4.12) has at least one T-periodic solution, and if $s \leq 0$, then equation (4.12) has no T-periodic solution.

Proof We only consider the first case; the other case can be considered similarly. Using the limit $\lim _{x \rightarrow-\infty} g(x)=+\infty$, we know that for any $s>0$, there exists a constant $X_{0}>0$ such that

$$
g(x)>s, \quad \forall x<-X_{0} .
$$

Similarly, using $\lim _{x \rightarrow+\infty} g(x)=0$, we know that for any $s>0$, there exists a constant $X_{0}^{\prime}>0$ such that

$$
g(x)<s, \quad \forall x>X_{0}^{\prime} .
$$

Let $\gamma=F_{0}+\left(g_{0}+\|p\|_{\infty}+s\right) T+1$, where

$$
g_{0}=\max _{x \in\left[-2 a T-X_{0}, X_{0}^{\prime}+2 a T\right]}|g(x)|, \quad F_{0}=\max _{x \in\left[-2 a T-X_{0}, X_{0}^{\prime}+2 a T\right]}|F(x)| .
$$

Now consider the rectangle

$$
\mathcal{D}=\left\{(x, y) \in \mathbb{R}^{2}:-a T-X_{0} \leq x \leq X_{0}^{\prime}+a T,-\gamma \leq y \leq \gamma\right\} .
$$

By the same arguments as in the proof of Theorem 4.4 or Theorem 4.5 we can verify that $\mathcal{F}$ (defined in the proof of Theorem 4.4) satisfies the bend-twist condition on $\mathcal{D}$ and obtain a fixed point of the Poincaré mapping, which is corresponding to a $T$-periodic solution.

If $s \leq 0$, then by integrating from 0 to $T$ both sides of the second equality of (4.19) we have

$$
\begin{aligned}
y(T)-y(0) & =\int_{0}^{T}[-g(x(\tau))+s] \mathrm{d} \tau+\int_{0}^{T} p(\tau) \mathrm{d} \tau \\
& =\int_{0}^{T}[-g(x(\tau))+s] \mathrm{d} \tau<0,
\end{aligned}
$$

which implies that equation (4.12) has no $T$-periodic solution. 
Using Theorem 4.6, we have the following example.

Example 4.4 Let $e(t) \in \widetilde{C}_{T}, b, c, q>0, p>1$. Then the problem

$$
\begin{aligned}
& \left(\frac{x^{\prime}}{\sqrt{1-x^{\prime 2}}}\right)^{\prime}+c|x|^{q} x^{\prime}+b \exp \left(|x|^{p-1} x\right)=e(t)+s, \\
& x(0)-x(T)=0=x^{\prime}(0)-x^{\prime}(T),
\end{aligned}
$$

has at least one solution if $s>0$ and has no solution if $s \leq 0$.

\section{Competing interests}

The authors declare that they have no competing interests.

\section{Authors' contributions}

All the authors contributed equally and significantly in writing this article. All the authors read and approved the final manuscript.

\section{Author details}

'School of Mathematical Sciences, Soochow University, Suzhou, 215006, China. ${ }^{2}$ School of Information and Technology, Shandong Agricultural University, Taian, 271018, China. ${ }^{3}$ School of Mathematics and Computing Sciences, Guilin University of Electronic Technology, Guilin, 541002, China.

\section{Acknowledgements}

This work is supported by the National Natural Science Foundation (Nos. 11271277, 11301106) and Guangxi Natural Science Foundation (Nos. 2014GXNSFBA118017, 2014GXNSFAA118004).

\section{Received: 28 August 2015 Accepted: 4 February 2016 Published online: 15 February 2016}

\section{References}

1. Reiser, M: Theory and Design of Charged Particle Beams. Wiley-VCH, Weinheim (2008)

2. Moser, J, Zehnder, E: Notes on Dynamical Systems. Am. Math. Soc., New York (2005)

3. Roberts, CS, Buchsbaum, S: Motion of a charged particle in a constant magnetic field and a transverse electromagnetic wave propagating along the field. Phys. Rev. A 135, 381-389 (1964)

4. Mourou, GA, Tajima, T, Bulanov, SV: Optics in the relativistic regime. Rev. Mod. Phys. 78(2), 309-371 (2006)

5. Allison, W, Cobb, J: Relativistic charged particle identification by energy loss. Annu. Rev. Nucl. Part. Sci. 30(1), 253-298 (1980)

6. Kirk, J, Schneider, P: On the acceleration of charged particles at relativistic shock fronts. Astrophys. J. 315(1), 425-433 (1987)

7. Schlickeiser, R, Lerche, I: Nonlinear radiative cooling of relativistic particles under equipartition conditions. Astron. Astrophys. 476(1), 1-8 (2007)

8. Bereanu, C, Mawhin, J: Existence and multiplicity results for some nonlinear problems with singular $\phi$-Laplacian. J. Differ. Equ. 243(2), 536-557 (2007)

9. Bereanu, C, Mawhin, J: Multiple periodic solutions of ordinary differential equations with bounded nonlinearities and $\phi$-Laplacian. NoDEA Nonlinear Differ. Equ. Appl. 15(1), 159-168 (2008)

10. Bereanu, C, Mawhin, J: Boundary value problems for second-order nonlinear difference equations with discrete $\phi$-Laplacian and singular $\phi$. J. Differ. Equ. Appl. 14(10), 1099-1118 (2008)

11. Bereanu, C, Mawhin, J: Nonhomogeneous boundary value problems for some nonlinear equations with singular $\phi$-Laplacian. J. Math. Anal. Appl. 352(1), 218-233 (2009)

12. Bereanu, C, Mawhin, J: Boundary value problems for some nonlinear systems with singular $\phi$-Laplacian. J. Fixed Point Theory Appl. 4(1), 57-75 (2008)

13. Bereanu, C, Jebelean, $P$, Mawhin, J: Non-homogeneous boundary value problems for ordinary and partial differential equations involving singular $\phi$-Laplacians. Mat. Contemp. 36, 51-65 (2009)

14. Brezis, H, Mawhin, J: Periodic solutions of the forced relativistic pendulum. Differ. Integral Equ. 23(9), $801-810$ (2010)

15. Bereanu, C, Jebelean, P, Mawhin, J: Periodic solutions of pendulum-like perturbations of singular and bounded $\phi$-Laplacians. J. Dyn. Differ. Equ. 22(3), 463-471 (2010)

16. Cid, JÁ, Torres, PJ: Solvability for some boundary value problems with $\phi$-Laplacian operators. Discrete Contin. Dyn. Syst., Ser. A 23(3), 727-732 (2009)

17. O'Regan, D, Perán, J: One dimensional $\phi$-Laplacian functional equations. J. Math. Anal. Appl. 371(1), 177-183 (2010)

18. Bereanu, C, Torres, PJ: Existence of at least two periodic solutions of the forced relativistic pendulum. Proc. Am. Math. Soc. 140, 2713-2719 (2012)

19. Coelho, I, Corsato, C, Obersnel, F, Omari, P: Positive solutions of the Dirichlet problem for the one-dimensional Minkowski-curvature equation. Adv. Nonlinear Stud. 12(3), 621-638 (2012)

20. Liu, Q, Qian, D, Chu, B: Nonlinear systems with singular vector $\phi$-Laplacian under the Hartman-type condition. Nonlinear Anal. TMA 74(8), 2880-2886 (2011)

21. Goldstein, H: Classical Mechanics. Addison-Wesley, Cambridge (1951)

22. Kim, JH, Lee, HW: Relativistic chaos in the driven harmonic oscillator. Phys. Rev. E 51(2), 1579-1581 (1995) 
23. Torres, P: Periodic oscillations of the relativistic pendulum with friction. Phys. Lett. A 372(42), 6386-6387 (2008)

24. Torres, P: Nondegeneracy of the periodically forced Liénard differential equation with $\varphi$-Laplacian. Commun Contemp. Math. 13(2), 283-292 (2011)

25. Liu, Q, Wang, C, Wang, Z: On Littlewood's boundedness problem for relativistic oscillators with anharmonic potentials. J. Differ. Equ. 257(12), 4542-4571 (2014)

26. Brezis, H, Mawhin, J: Periodic solutions of Lagrangian systems of relativistic oscillators. Commun. Appl. Anal. 15(2), 235-250 (2011)

27. Mawhin, J: Multiplicity of solutions of variational systems involving $\varphi$-Laplacians with $\operatorname{singular} \varphi$ and periodic nonlinearities. Discrete Contin. Dyn. Syst. 32, 4015-4026 (2012)

28. Bereanu, C, Jebelean, P, Mawhin, J: Radial solutions for Neumann problems involving mean curvature operators in Euclidean and Minkowski spaces. Math. Nachr. 283(3), 379-391 (2010)

29. Jebelean, P, Mawhin, J, Şerban, C: Multiple periodic solutions for perturbed relativistic pendulum systems. Proc. Am. Math. Soc. 143(7), 3029-3039 (2015)

30. Rouche, N, Mawhin, JL: Ordinary Differential Equations: Stability and Periodic Solutions. Surveys and Reference Works in Mathematics, vol. 5. Pitman Advanced Publishing Program, Boston (1980)

31. Mawhin, J: Variations on Poincaré-Miranda's theorem. Adv. Nonlinear Stud. 13, 209-217 (2013)

32. Ding, T, lannacci, R, Zanolin, F: Existence and multiplicity results for periodic solutions of semilinear Duffing equations J. Differ. Equ. 105(2), 364-409 (1993)

33. Sedziwy, S: On certain results of C. Bereanu and J. Mawhin. Bull. Belg. Math. Soc. Simon Stevin 19(4), 649-653 (2012)

\section{Submit your manuscript to a SpringerOpen ${ }^{\odot}$ journal and benefit from:}

- Convenient online submission

Rigorous peer review

- Immediate publication on acceptance

- Open access: articles freely available online

- High visibility within the field

- Retaining the copyright to your article 